\title{
A Calabi-Yau threefold with non-Abelian fundamental group
}

\author{
Arnaud Beauville
}

The aim of this note is to answer a question of I. Dolgachev by constructing a Calabi-Yau threefold whose fundamental group is the quaternionic group $\mathrm{H}$ with 8 elements. The construction is very reminiscent of Reid's unpublished construction of a surface with $p_{g}=0, \mathrm{~K}^{2}=2$ and $\pi_{1}=\mathrm{H}$; I explain below the link between the two problems.

\section{The example}

Let $\mathrm{H}=\{ \pm 1, \pm i, \pm j, \pm k\}$ be the quaternionic group, and $\mathrm{V}$ its regular representation. We denote by $\widehat{\mathrm{H}}$ the group of characters $\chi: \mathrm{H} \rightarrow \mathbf{C}^{*}$; it is isomorphic to $\mathbf{Z}_{2} \times \mathbf{Z}_{2}$. The group $\mathrm{H}$ acts on $\mathbf{P}(\mathrm{V})^{1}$ and on $\mathbf{S}^{2} \mathrm{~V}$; for each $\chi \in \widehat{\mathrm{H}}$, we denote by $\left(\mathrm{S}^{2} \mathrm{~V}\right)_{\chi}$ the eigensubspace of $\mathrm{S}^{2} \mathrm{~V}$ with respect to $\chi$, i.e. the space of quadratic forms $\mathrm{Q}$ on $\mathbf{P}(\mathrm{V})$ such that $h \cdot \mathrm{Q}=\chi(h) \mathrm{Q}$ for all $h \in \mathrm{H}$.

Theorem.- For each $\chi \in \widehat{\mathrm{H}}$, let $\mathrm{Q}_{\chi}$ be a general element of $\left(\mathrm{S}^{2} \mathrm{~V}\right)_{\chi}$. The subvariety $\widetilde{\mathrm{X}}$ of $\mathbf{P}(\mathrm{V})$ defined by the 4 equations $\mathrm{Q}_{\chi}=0(\chi \in \widehat{\mathrm{H}})$ is a smooth threefold, on which the group $\mathrm{H}$ acts freely. The quotient $\mathrm{X}:=\widetilde{\mathrm{X}} / \mathrm{H}$ is a Calabi-Yau threefold with $\pi_{1}(\mathrm{X})=\mathrm{H}$.

Let me observe first that the last assertion is an immediate consequence of the others. Indeed, since $\widetilde{\mathrm{X}}$ is a Calabi-Yau threefold, one has $h^{1,0}(\widetilde{\mathrm{X}})=h^{2,0}(\widetilde{\mathrm{X}})=$ $\chi\left(\mathcal{O}_{\widetilde{\mathrm{X}}}\right)=0$, hence $h^{1,0}(\mathrm{X})=h^{2,0}(\mathrm{X})=\chi\left(\mathcal{O}_{\mathrm{X}}\right)=0$. This implies $h^{1,0}(\mathrm{X})=1$, so there exists a nonzero holomorphic 3 -form $\omega$ on $\mathrm{X}$; since its pull-back to $\widetilde{\mathrm{X}}$ is everywhere nonzero, $\omega$ has the same property, hence $\mathrm{X}$ is a Calabi-Yau threefold. Finally $\widetilde{\mathrm{X}}$ is a complete intersection in $\mathbf{P}(\mathrm{V})$, hence simply connected by Lefschetz' theorem, so the fundamental group of $\mathrm{X}$ is isomorphic to $\mathrm{H}$.

So the problem is to prove that $\mathrm{H}$ acts freely and $\widetilde{\mathrm{X}}$ is smooth. We will need to write down explicitely the elements of $\left(\mathrm{S}^{2} \mathrm{~V}\right)_{\chi}$. As a $\mathrm{H}$-module, $\mathrm{V}$ is the direct sum of the 4 one-dimensional representations of $\mathrm{H}$ and twice the irreducible two-dimensional representation $\rho$. Thus there exists a system of homogeneous coordinates $\left(\mathrm{X}_{1}, \mathrm{X}_{\alpha}, \mathrm{X}_{\beta}, \mathrm{X}_{\gamma} ; \mathrm{Y}, \mathrm{Z} ; \mathrm{Y}^{\prime}, \mathrm{Z}^{\prime}\right)$ such that

$g \cdot\left(\mathrm{X}_{1}, \mathrm{X}_{\alpha}, \mathrm{X}_{\beta}, \mathrm{X}_{\gamma} ; \mathrm{Y}, \mathrm{Z} ; \mathrm{Y}^{\prime}, \mathrm{Z}^{\prime}\right)=\left(\mathrm{X}_{1}, \alpha(g) \mathrm{X}_{\alpha}, \beta(g) \mathrm{X}_{\beta}, \gamma(g) \mathrm{X}_{\gamma} ; \rho(g)(\mathrm{Y}, \mathrm{Z}) ; \rho(g)\left(\mathrm{Y}^{\prime}, \mathrm{Z}^{\prime}\right)\right)$.

To be more precise, I denote by $\alpha$ (resp. $\beta$, resp. $\gamma$ ) the nontrivial character which is +1 on $i$ (resp. $j$, resp. $k$ ), and I take for $\rho$ the usual representation via

$\overline{1}$ I use Grothendieck's notation, i.e. $\mathbf{P}(\mathrm{V})$ is the space of hyperplanes in V. 
Pauli matrices:

$\rho(i)(\mathrm{Y}, \mathrm{Z})=(\sqrt{-1} \mathrm{Y},-\sqrt{-1} \mathrm{Z}) \quad \rho(j)(\mathrm{Y}, \mathrm{Z})=(-\mathrm{Z}, \mathrm{Y}) \quad \rho(k)(\mathrm{Y}, \mathrm{Z})=(-\sqrt{-1} \mathrm{Z},-\sqrt{-1} \mathrm{Y})$

Then the general element $\mathrm{Q}_{\chi}$ of $\left(\mathrm{S}^{2} \mathrm{~V}\right)_{\chi}$ can be written

$$
\begin{aligned}
& \mathrm{Q}_{1}=t_{1}^{1} \mathrm{X}_{1}^{2}+t_{2}^{1} \mathrm{X}_{\alpha}^{2}+t_{3}^{1} \mathrm{X}_{\beta}^{2}+t_{4}^{1} \mathrm{X}_{\gamma}^{2}+t_{5}^{1}\left(\mathrm{YZ}^{\prime}-\mathrm{Y}^{\prime} \mathrm{Z}\right) \\
& \mathrm{Q}_{\alpha}=t_{1}^{\alpha} \mathrm{X}_{1} \mathrm{X}_{\alpha}+t_{2}^{\alpha} \mathrm{X}_{\beta} \mathrm{X}_{\gamma}+t_{3}^{\alpha} \mathrm{YZ}+t_{4}^{\alpha} \mathrm{Y}^{\prime} \mathrm{Z}^{\prime}+t_{5}^{\alpha}\left(\mathrm{YZ}^{\prime}+\mathrm{ZY}^{\prime}\right) \\
& \mathrm{Q}_{\beta}=t_{1}^{\beta} \mathrm{X}_{1} \mathrm{X}_{\beta}+t_{2}^{\beta} \mathrm{X}_{\alpha} \mathrm{X}_{\gamma}+t_{3}^{\beta}\left(\mathrm{Y}^{2}+\mathrm{Z}^{2}\right)+t_{4}^{\beta}\left(\mathrm{Y}^{\prime 2}+\mathrm{Z}^{\prime 2}\right)+t_{5}^{\beta}\left(\mathrm{YY}^{\prime}+\mathrm{ZZ}^{\prime}\right) \\
& \mathrm{Q}_{\gamma}=t_{1}^{\gamma} \mathrm{X}_{1} \mathrm{X}_{\gamma}+t_{2}^{\gamma} \mathrm{X}_{\alpha} \mathrm{X}_{\beta}+t_{3}^{\gamma}\left(\mathrm{Y}^{2}-\mathrm{Z}^{2}\right)+t_{4}^{\gamma}\left(\mathrm{Y}^{\prime 2}-\mathrm{Z}^{\prime 2}\right)+t_{5}^{\gamma}\left(\mathrm{YY}^{\prime}-\mathrm{ZZ}^{\prime}\right) .
\end{aligned}
$$

For $\mathbf{t}:=\left(t_{i}^{\chi}\right)$ fixed, let $\mathcal{X}_{\mathbf{t}}$ be the subvariety of $\mathbf{P}(\mathrm{V})$ defined by the equations $\mathrm{Q}_{\chi}=0$. Let us check first that the action of $\mathrm{H}$ on $\mathcal{X}_{\mathbf{t}}$ is fixed point free for $\mathbf{t}$ general enough. Since a point fixed by an element $h$ of $\mathrm{H}$ is also fixed by $h^{2}$, it is sufficient to check that the element $-1 \in \mathrm{H}$ acts without fixed point, i.e. that $\mathcal{X}_{\mathbf{t}}$ does not meet the linear spaces $\mathrm{L}_{+}$and $\mathrm{L}_{-}$defined by $\mathrm{Y}=\mathrm{Z}=\mathrm{Y}^{\prime}=\mathrm{Z}^{\prime}=0$ and $\mathrm{X}_{1}=\mathrm{X}_{\alpha}=\mathrm{X}_{\beta}=\mathrm{X}_{\gamma}=0$ respectively.

Let $x=\left(0,0,0,0 ; \mathrm{Y}, \mathrm{Z} ; \mathrm{Y}^{\prime}, \mathrm{Z}^{\prime}\right) \in \mathcal{X}_{\mathbf{t}} \cap \mathrm{L}_{-}$. One of the coordinates, say $\mathrm{Z}$, is nonzero; since $\mathrm{Q}_{1}(x)=0$, there exists $k \in \mathbf{C}$ such that $\mathrm{Y}^{\prime}=k \mathrm{Y}, \mathrm{Z}^{\prime}=k \mathrm{Z}$. Substituting in the equations $\mathrm{Q}_{\alpha}(x)=\mathrm{Q}_{\beta}(x)=\mathrm{Q}_{\gamma}(x)=0$ gives

$\left(t_{3}^{\alpha}+t_{5}^{\alpha} k+t_{4}^{\alpha} k^{2}\right) \mathrm{YZ}=\left(t_{3}^{\beta}+t_{5}^{\beta} k+t_{4}^{\beta} k^{2}\right)\left(\mathrm{Y}^{2}+\mathrm{Z}^{2}\right)=\left(t_{3}^{\alpha}+t_{5}^{\alpha} k+t_{4}^{\alpha} k^{2}\right)\left(\mathrm{Y}^{2}-\mathrm{Z}^{2}\right)=0$

which has no nonzero solutions for a generic choice of $\mathbf{t}$.

Now let $x=\left(\mathrm{X}_{1}, \mathrm{X}_{\alpha}, \mathrm{X}_{\beta}, \mathrm{X}_{\gamma} ; 0,0 ; 0,0\right) \in \mathcal{X}_{\mathbf{t}} \cap \mathrm{L}_{+}$. As soon as the $t_{i}^{\chi}$ 's are nonzero, two of the $\mathrm{X}$-coordinates cannot vanish, otherwise all the coordinates would be zero. Expressing that $\mathrm{Q}_{\beta}=\mathrm{Q}_{\gamma}=0$ has a nontrivial solution in $\left(\mathrm{X}_{\beta}, \mathrm{X}_{\gamma}\right)$ gives $\mathrm{X}_{\alpha}^{2}$ as a multiple of $\mathrm{X}_{1}^{2}$, and similarly for $\mathrm{X}_{\beta}^{2}$ and $\mathrm{X}_{\gamma}^{2}$. But then $\mathrm{Q}_{1}(x)=0$ is impossible for a general choice of $\mathbf{t}$.

Now we want to prove that $\mathcal{X}_{\mathbf{t}}$ is smooth for $\mathbf{t}$ general enough. Let $\mathcal{Q}=$ $\oplus_{\widehat{\widehat{H}}}\left(\mathrm{~S}^{2} \mathrm{~V}\right)_{\chi}$; then $\mathbf{t}:=\left(t_{i}^{\chi}\right)$ is a system of coordinates on $\mathcal{Q}$. The equations $\mathrm{Q}_{\chi}=0$ $\chi \in \widehat{\mathrm{H}}$

define a subvariety $\mathcal{X}$ in $\mathcal{Q} \times \mathbf{P}(\mathrm{V})$, whose fibre above a point $\mathbf{t} \in \mathcal{Q}$ is $\mathcal{X}_{\mathbf{t}}$. Consider the second projection $p: \mathcal{X} \rightarrow \mathbf{P}(\mathrm{V})$. For $x \in \mathbf{P}(\mathrm{V})$, the fibre $p^{-1}(x)$ is the linear subspace of $\mathcal{Q}$ defined by the vanishing of the $\mathrm{Q}_{\chi}$ 's, viewed as linear forms in $\mathbf{t}$. These forms are clearly linearly independent as soon as they do not vanish. In other words, if we denote by $B_{\chi}$ the base locus of the quadrics in $\left(S^{2} V\right)_{\chi}$ and put $\mathrm{B}=\cup \mathrm{B}_{\chi}$, the map $p: \mathcal{X} \rightarrow \mathbf{P}(\mathrm{V})$ is a vector bundle fibration above $\mathbf{P}(\mathrm{V})-\mathrm{B}$; in particular $\mathcal{X}$ is non-singular outside $p^{-1}(\mathrm{~B})$. Therefore it is enough to prove that $\mathcal{X}_{\mathbf{t}}$ is smooth at the points of $\mathrm{B} \cap \mathcal{X}_{\mathbf{t}}$. 
Observe that an element $x$ in $\mathrm{B}$ has two of its $\mathrm{X}$-coordinates zero. Since the equations are symmetric in the $\mathrm{X}$-coordinates we may assume $\mathrm{X}_{\beta}=\mathrm{X}_{\gamma}=0$. Then the Jacobian matrix $\left(\frac{\partial \mathrm{Q}_{\chi}}{\partial \mathrm{X}_{\psi}}(x)\right)$ takes the form

$$
\left(\begin{array}{cccc}
2 t_{1}^{1} \mathrm{X}_{1} & 2 t_{2}^{1} \mathrm{X}_{\alpha} & 0 & 0 \\
t_{1}^{\alpha} \mathrm{X}_{\alpha} & t_{1}^{\alpha} \mathrm{X}_{1} & 0 & 0 \\
0 & 0 & t_{1}^{\beta} \mathrm{X}_{1} & t_{2}^{\beta} \mathrm{X}_{\alpha} \\
0 & 0 & t_{2}^{\gamma} \mathrm{X}_{\alpha} & t_{1}^{\gamma} \mathrm{X}_{1}
\end{array}\right)
$$

For generic $\mathbf{t}$ this matrix is of rank 4 except when all the $\mathrm{X}$-coordinates of $x$ vanish; but we have seen that this is impossible when $\mathbf{t}$ is general enough.

\section{Some comments}

As mentioned in the introduction, the construction is inspired by Reid's example of a surface of general type with $p_{g}=0, \mathrm{~K}^{2}=2, \pi_{1}=\mathrm{H}[\mathrm{R}]$. This is more than a coincidence. In fact, let $\widetilde{\mathrm{S}}$ be the hyperplane section $\mathrm{X}_{1}=0$ of $\widetilde{\mathrm{X}}$. It is stable under the action of $\mathrm{H}$ (so that $\mathrm{H}$ acts freely on $\widetilde{\mathrm{S}}$ ), and one can prove as above that it is smooth for a generic choice of the parameters. The surface $\mathrm{S}:=\widetilde{\mathrm{S}} / \mathrm{H}$ is a Reid surface, embedded in $\mathrm{X}$ as an ample divisor, with $h^{0}\left(\mathrm{X}, \mathcal{O}_{\mathrm{X}}(\mathrm{S})\right)=1$.

In general, let us consider a Calabi-Yau threefold $\mathrm{X}$ which contains a rigid ample surface - i.e. a smooth ample divisor $\mathrm{S}$ such that $h^{0}\left(\mathcal{O}_{\mathrm{X}}(\mathrm{S})\right)=1$. Put $\mathrm{L}:=\mathcal{O}_{\mathrm{X}}(\mathrm{S})$. Then $\mathrm{S}$ is a minimal surface of general type (because $\mathrm{K}_{\mathrm{S}}=\mathrm{L}_{\mid \mathrm{S}}$ is ample); by the Lefschetz theorem, the natural map $\pi_{1}(\mathrm{~S}) \rightarrow \pi_{1}(\mathrm{X})$ is an isomorphism. Because of the exact sequence

$$
0 \rightarrow \mathcal{O}_{\mathrm{X}} \longrightarrow \mathrm{L} \longrightarrow \mathrm{K}_{\mathrm{S}} \rightarrow 0
$$

the geometric genus $p_{g}(\mathrm{~S}):=h^{0}\left(\mathrm{~K}_{\mathrm{S}}\right)$ is zero.

One has $\mathrm{K}_{\mathrm{S}}^{2}=\mathrm{L}^{3}$; the Riemann-Roch theorem on $\mathrm{X}$ yields

$$
1=h^{0}(\mathrm{~L})=\frac{\mathrm{L}^{3}}{6}+\frac{\mathrm{L} \cdot c_{2}}{12} ;
$$

by Miyaoka theorem [Mi] one has $\mathrm{L} \cdot c_{2}>0$ (the strict inequality requires playing around a little bit with the index theorem), hence $\mathrm{K}_{\mathrm{S}}^{2} \leq 5$.

With a few exceptions, the possible fundamental groups of surfaces with $p_{g}=0$ and $\mathrm{K}_{\mathrm{S}}^{2}=1$ or 2 are known (see [B-P-V] for an overview). In the case $\mathrm{K}_{\mathrm{S}}^{2}=1$, the fundamental group is cyclic of order $\leq 5$; if $\mathrm{K}_{\mathrm{S}}^{2}=2$, it is of order $\leq 9$; moreover the dihedral group $\mathrm{D}_{8}$ cannot occur. I believe that the symmetric group $\mathfrak{S}_{3}$ cannot occur either, though I do not think the proof has been written down. If this is true, the quaternionic group $\mathrm{H}$ is the only non-Abelian group which occurs in this range. 
On the other hand, little is known about surfaces with $p_{g}=0$ and $\mathrm{K}_{\mathrm{S}}^{2}=3,4$ or 5. Inoue has constructed examples with $\pi_{1}=\mathrm{H} \times\left(\mathbf{Z}_{2}\right)^{n}$, with $n=\mathrm{K}^{2}-2$ (loc. cit.); I do not know if they can appear as rigid ample surfaces in a CalabiYau threefold.

Let us denote by $\widetilde{\mathrm{X}}$ the universal cover of $\mathrm{X}$, by $\widetilde{\mathrm{L}}$ the pull back of $\mathrm{L}$ to $\widetilde{\mathrm{X}}$, and by $\rho$ the representation of $\mathrm{G}$ on $\mathrm{H}^{0}(\widetilde{\mathrm{X}}, \widetilde{\mathrm{L}})$. One has $\operatorname{Tr} \rho(g)=0$ for $g \neq 1$ by the holomorphic Lefschetz formula, and $\operatorname{Tr} \rho(1)=\chi(\widetilde{\mathrm{L}})=|\mathrm{G}| \chi(\mathrm{L})=|\mathrm{G}|$. Therefore $\rho$ is isomorphic to the regular representation. Looking at the list in loc. cit. one gets a few examples of this situation, for instance:

$-\mathrm{G}=\mathbf{Z}_{5}, \widetilde{\mathrm{X}}=$ a quintic hypersurface in $\mathbf{P}^{4}$;

- $\mathrm{G}=\left(\mathbf{Z}_{2}\right)^{3}$ or $\mathbf{Z}_{\mathbf{4}} \times \mathbf{Z}_{2}, \widetilde{\mathrm{X}}=$ an intersection of 4 quadrics in $\mathbf{P}^{7}$ as above;

$-\mathrm{G}=\mathbf{Z}_{3} \times \mathbf{Z}_{3}, \widetilde{X}=$ a hypersurface of bidegree $(3,3)$ in $\mathbf{P}^{2} \times \mathbf{P}^{2}$.

Of course when looking for Calabi-Yau threefolds with interesting $\pi_{1}$ there is no reason to assume that it contains an ample rigid surface. Observe however that if we want to use the preceding method, i.e. find a projective space $\mathbf{P}(\mathrm{V})$ with an action of $\mathrm{G}$ and a smooth invariant linearly normal Calabi-Yau threefold $\widetilde{\mathrm{X}} \subset \mathbf{P}(\mathrm{V})$, then the line bundle $\mathcal{O}_{\widetilde{\mathrm{X}}}(1)$ will be the pull-back of an ample line bundle $\mathrm{L}$ on $\mathrm{X}$, and by the above argument the representation of $\mathrm{G}$ on $\mathrm{V}$ will be $h^{0}(\mathrm{~L})$ times the regular representation. This leaves little hope to find an invariant CalabiYau threefold when the product $h^{0}(\mathrm{~L})|\mathrm{G}|$ becomes large.

\section{REFERENCES}

[B-P-V] W. Barth, C. Peters, A. Van de Ven: Compact complex surfaces. Ergebnisse der Math., Springer-Verlag (1984).

[Mi] Y. MiYaOKa: The Chern classes and Kodaira dimension of a minimal variety. Adv. Studies in Pure Math. 10, 449-476, Kinokuniya-North Holland (1987).

[R] M. ReID: Surfaces with $p_{g}=0, \mathrm{~K}^{2}=2$. Unpublished manuscript (1979).

A. Beauville

URA $752 \mathrm{du}$ CNRS

Mathématiques - Bât. 425

Université Paris-Sud

91405 Orsay Cedex, France 